\title{
DESIGN OF OVERSAMPLED UNIFORM DFT FILTER BANKS WITH DELAY SPECIFICATION USING QUADRATIC OPTIMIZATION
}

\author{
J. M. de Haan, N. Grbić, I. Claesson \\ Blekinge Institute of Technology \\ Department of Telecommunications \\ and Signal Processing \\ 37225 Ronneby, Sweden
}

\author{
S. Nordholm \\ Australian Telecommunications \\ Research Institute \\ Curtin University \\ Perth, Australia
}

\begin{abstract}
Subband adaptive filters have been proposed to avoid the drawbacks of slow convergence and high computational complexity associated with time domain adaptive filters. Subband processing introduces transmission delays caused by the filter bank and signal degradations due to aliasing effects. One efficient way to reduce the aliasing effects is to allow a higher sample rate than critically needed in the subbands and thus reduce subband signal degradation. We suggest a design method, for uniform DFT filter banks with any oversampling factor, where the total filter bank group delay may be specified, and where the aliasing and magnitude/phase distortions are minimized.
\end{abstract}

\section{INTRODUCTION}

Subband adaptive filtering has arised as an alternative for conventional time domain adaptive filtering, [1]. The main reason is the reduction in computational complexity and the increase in convergence speed for the adaptive algorithm, achieved by dividing the algorithm into subbands, [2]. The computational savings comes from the fact that time domain convolution becomes decoupled in the subbands, at a lower sample rate, [3].

Subband analysis and synthesis is often performed using multirate filter banks, [4]. Non-ideal filters in the filter bank cause aliasing of the subband signals. This aliasing can be cancelled in the synthesis bank when certain conditions are met by the synthesis filters and in the subband processing. However, even if aliasing distortion in the filter bank output is cancelled in this way, the inband aliasing is still present in the subband adaptive filter input signals and, consequently, the adaptive filters are perturbed and the overall performance of the system is reduced, [5].

Several solutions to the subband filtering problem have been suggested in literature. Non-critical decimation has been suggested in [1], where filter bank delay aspects, and amplitude distortions, have not especially been taken into consideration. The use of cross filters, [5], has been suggested to explicitly filter out the aliasing components. A delayless structure has been proposed in [6], where the actual filtering is performed in the time domain, with consequences of higher computational complexity. The computational complexity also increases significantly with cross band filters.
We use a uniform DFT-modulated FIR filter bank for the subband transformations. Modulated filter banks provide a computationally efficient implementation, due to the polyphase implementation [4], and great design simplicity. The main contribution in this paper is the suggested design method, where the filter bank response error and the inband and output aliasing errors are minimized simultaneously, while the total filter bank group-delay is pre-specified. The influence of the filter bank performance is evaluated on an RLS subband beamformer [7].

\section{THE UNIFORM DFT MODULATED FILTER BANK}

In this section we will derive an input-output expression for analysis-synthesis DFT filter banks with arbitrary decimation factor. Two sets of $M$ filters, $H_{m}(z)$ and $G_{m}(z)$, form a uniform DFT analysis filter bank and synthesis filter bank, respectively, when they are related to prototype filters, $H(z)$ and $G(z)$, as

$$
\begin{array}{r}
H_{m}(z)=H\left(z W_{M}^{m}\right)=\mathbf{h}^{T} \phi\left(z W_{M}^{m}\right) \\
G_{m}(z)=G\left(z W_{M}^{m}\right)=\mathbf{g}^{T} \phi\left(z W_{M}^{m}\right) \\
\text { for } m=0, \ldots, M-1
\end{array}
$$

where $W_{M}=e^{-j 2 \pi / M}, \mathbf{h}=\left[h(0), \ldots, h\left(L_{h}-1\right)\right]^{T}, \mathbf{g}=$ $\left[g(0), \ldots, g\left(L_{g}-1\right)\right]^{T}$ and $\phi(z)=\left[1, z^{-1}, \ldots, z^{-(L-1)}\right]^{T}$. Each subband signal is decimated by a factor $D$. An efficient implementation of such a filter bank is given in [8]. For simplicity of derivation we study the direct form realization of the filter banks given in Fig. 1. The input signal

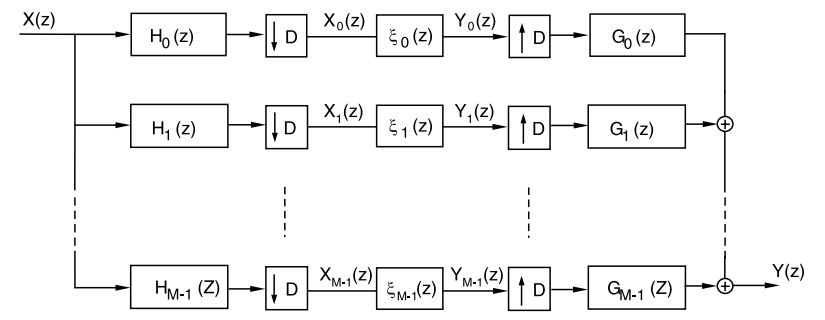

Figure 1: Direct form realization for analysis and synthesis filter banks. 
$X(z)$ is filtered by the analysis filters $H_{m}(z)$ and decimated by the factor $D$ according to

$$
\begin{gathered}
X_{m}(z)=\frac{1}{D} \sum_{l=0}^{D-1} H\left(z^{\frac{1}{D}} W_{M}^{m} W_{D}^{l}\right) X\left(z^{\frac{1}{D}} W_{D}^{l}\right) \\
m=0, \cdots, M-1
\end{gathered}
$$

where $W_{D}=e^{-j 2 \pi / D}$. In the synthesis filter bank, the subband signals $Y_{m}$ are interpolated by the interpolation factor $D$, filtered by the synthesis filters $G_{m}(z)$ and then added together to form the output signal

$$
Y(z)=\frac{1}{D} \sum_{l=0}^{D-1} X\left(z W_{D}^{l}\right) \sum_{m=0}^{M-1} \xi_{m}\left(z^{D}\right) H\left(z W_{M}^{m} W_{D}^{l}\right) G\left(z W_{M}^{m}\right)
$$

Here $\xi_{m}(z)$ is the application dependent filtering operation in subband no. $m$.

\section{ANALYSIS FILTER BANK DESIGN}

Since the analysis filters are related to a single prototype analysis filter according to Eq. (1), the analysis filter bank design problem reduces to the design of a single filter. The ideal prototype analysis filter is a low pass filter filter with cut-off frequency $\omega_{p}=\pi / M$. Since FIR filters are not ideally frequency selective, approximations need to be made. The analysis prototype filter will be designed by defining a passband region in which the filter response should be flat, while minimizing the inband aliasing error. By minimizing the inband aliasing, the prototype filter will obtain low-pass characteristics since this is similar to maximizing stop band attenuation.

The passband response error in the passband region $\Omega_{p}=\left[-\omega_{p}, \omega_{p}\right]$ is defined as

$$
\varepsilon_{P}(\mathbf{h})=\frac{1}{2 \omega_{p}} \int_{-\omega_{p}}^{\omega_{p}}\left|H\left(e^{j \omega}\right)-H_{d}\left(e^{j \omega}\right)\right|^{2} d \omega
$$

where $H_{d}(z)$ is the desired frequency response. The desired frequency response is

$$
H_{d}\left(e^{j \omega}\right)=e^{-j \omega \tau_{h}} \quad \omega \in \Omega_{p}
$$

where $\tau_{h}$ is the desired group delay of the analysis prototype filter and with that the desired delay of the analysis filter bank. With the analysis prototype filter written in terms of its impulse response, $H(z)=\mathbf{h}^{T} \phi(z)$, we substitute Eq. (5) into Eq. (4)

$$
\varepsilon_{P}(\mathbf{h})=\mathbf{h}^{T} \mathbf{A} \mathbf{h}-2 \mathbf{h}^{T} \mathbf{b}+1
$$

where

$$
\mathbf{A}=\frac{1}{2 \omega_{p}} \int_{-\omega_{p}}^{\omega_{p}} \phi\left(e^{j \omega}\right) \phi^{H}\left(e^{j \omega}\right) d \omega
$$

and

$$
\mathbf{b}=\frac{1}{2 \omega_{p}} \int_{-\omega_{p}}^{\omega_{p}} \operatorname{Re}\left\{e^{j \omega \tau_{h}} \phi\left(e^{j \omega}\right)\right\} d \omega .
$$

In order to minimize the inband aliasing we will define the Inband Aliasing Error and express it in terms of the analysis prototype filter. From Eq. (2), the sum of the inband aliasing terms $\mathcal{X}_{m}(z)$ in subband signal $X_{m}(z)$ is described by

$$
\mathcal{X}_{m}(z)=\frac{1}{D} \sum_{l=1}^{D-1} H_{0}\left(z^{\frac{1}{D}} W_{M}^{m} W_{D}^{l}\right) X\left(z^{\frac{1}{D}} W_{D}^{l}\right)
$$

for $m=0, \cdots, M-1$. In the ideal case with the ideal prototype filter, and thus with zero aliasing in the subband signals, $X_{m}(z)$, the frequency response part in each term is zero and thus $\mathcal{X}_{m}(z)=0$. In the non-ideal case with FIR filters we would like to minimize the energy in each term. Since the analysis filters are related by Eq. (1) it is sufficient to minimize the energy in the aliasing terms of the first subband $(m=0)$. The sum of the power magnitudes of the aliasing terms in the first subband is

$$
D_{0}\left(e^{j \omega}\right)=\frac{1}{D} \sum_{l=1}^{D-1}\left|H\left(e^{j \omega / D} W_{D}^{l}\right)\right|^{2}
$$

Subsequently, the inband aliasing error expressed in terms of the impulse response of the prototype analysis filter is

$$
\varepsilon_{D_{0}}(\mathbf{h})=\frac{1}{2 \pi} \int_{-\pi}^{\pi} D_{0}\left(e^{j \omega}\right) d \omega=\mathbf{h}^{T} \mathbf{C h}
$$

where the hermitian matrix $\mathbf{C}$ is

$$
\mathbf{C}=\frac{1}{2 \pi D} \sum_{l=1}^{D-1} \int_{-\pi}^{\pi} \phi\left(e^{j \omega / D} W_{D}^{l}\right) \phi^{H}\left(e^{j \omega / D} W_{D}^{l}\right) d \omega .
$$

The optimal analysis prototype filter with respect to minimal passband response error and minimal energy in the aliasing components is found by minimizing the sum of the passband response error in Eq. (6) and the inband aliasing error in Eq. (11)

$$
\begin{aligned}
\varepsilon_{t o t}(\mathbf{h}) & =\varepsilon_{P}(\mathbf{h})+\varepsilon_{D_{0}}(\mathbf{h}) \\
& =\mathbf{h}^{T}(\mathbf{A}+\mathbf{C}) \mathbf{h}-2 \mathbf{h}^{T} \mathbf{b}+1
\end{aligned}
$$

that is, through solving the linear equation system

$$
\mathbf{h}=\arg \min _{\mathbf{h}} \varepsilon_{t o t}(\mathbf{h})=(\mathbf{A}+\mathbf{C})^{-1} \mathbf{b} .
$$

\section{SYNTHESIS FILTER BANK DESIGN}

Given the analysis filter bank with an analysis prototype filter $H(z)$ designed as described in Section 3, we will design an optimal synthesis filter bank which minimizes the amplitude and phase distortion of the total filter bank system and also minimizes the output aliasing distortion.

The first part in the design of the synthesis filter bank is the minimization of amplitude and phase distortion. We will derive the System Response Error expressed in terms of the impulse reponses of the prototype filters $H(z)=\mathbf{h}^{T} \phi(z)$ and $G(z)=\mathbf{g}^{T} \phi(z)$. The system response error is defined by

$$
\varepsilon_{T}=\frac{1}{2 \pi} \int_{-\pi}^{\pi}\left|T\left(e^{j \omega}\right)-T_{d}\left(e^{j \omega}\right)\right|^{2} d \omega .
$$


From Eq. (3), we can express the total filter bank system response in terms of $\mathbf{h}$ and $\mathbf{g}$, with $\xi_{m}(z)=1, m=$ $0, \ldots, M-1$

$$
T(z)=\frac{1}{D} \sum_{l=0}^{D-1} \sum_{m=0}^{M-1} H\left(z W_{M}^{m} W_{D}^{l}\right) G\left(z W_{M}^{m}\right)=\mathbf{h}^{T} \mathbf{D}(z) \mathbf{g}
$$

where

$$
\mathbf{D}(z)=\frac{1}{D} \sum_{l=0}^{D-1} \sum_{m=0}^{M-1} \phi\left(z W_{M}^{m} W_{D}^{l}\right) \phi^{T}\left(z W_{M}^{m}\right) .
$$

The desired filter bank response is

$$
T_{d}\left(e^{j \omega}\right)=e^{-j \omega \tau_{d}}
$$

where $\tau_{d}$ is the desired filter bank delay. Substituting Eq. (18) and Eq. (16) into Eq. (15) yields

$$
\varepsilon_{T}(\mathbf{g})=\mathbf{g}^{T} \mathbf{E} \mathbf{g}-2 \mathbf{g}^{T} \mathbf{f}+1
$$

where

$$
\mathbf{E}=\frac{1}{2 \pi} \int_{-\pi}^{\pi} \mathbf{D}^{H}\left(e^{j \omega}\right) \mathbf{h}^{*} \mathbf{h}^{T} \mathbf{D}\left(e^{j \omega}\right) d \omega
$$

and

$$
\mathbf{f}=\frac{1}{2 \pi} \int_{-\pi}^{\pi} \operatorname{Re}\left\{e^{j \omega \tau_{d}} \mathbf{D}^{T}\left(e^{j \omega}\right) \mathbf{h}\right\} d \omega .
$$

From Eq. (3) we know that the aliasing terms $\mathcal{Y}(z)$ in the filter bank output signal $Y(z)$ are described by the sum of all repeated spectra

$$
\mathcal{Y}(z)=\frac{1}{D} \sum_{l=1}^{D-1} X\left(z W_{D}^{l}\right) \sum_{m=0}^{M-1} \xi_{m}\left(z^{D}\right) H\left(z W_{M}^{m} W_{D}^{l}\right) G\left(z W_{M}^{m}\right)
$$

In the ideal case, the aliasing terms in the output signal are zero, i.e. $\mathcal{Y}(z)=0$. In this case the prototype filters are such that the products of $H$ and $G$ in Eq. (22) are zero for all terms. In the non-ideal case we wish to minimize the energy in all aliasing terms. We define the sum of power magnitudes

$$
D\left(e^{j \omega}\right)=\frac{1}{D} \sum_{l=1}^{D-1} \sum_{m=0}^{M-1}\left|H\left(e^{j \omega} W_{M}^{m} W_{D}^{l}\right) G\left(e^{j \omega} W_{M}^{m}\right)\right|^{2} .
$$

We can rewrite Eq. (23) using the impulse responses $\mathbf{h}$ and g

$$
D\left(e^{j \omega}\right)=\frac{1}{D} \sum_{l=1}^{D-1} \sum_{m=0}^{M-1}\left|\mathbf{h}^{T} \boldsymbol{\Phi}_{m, l}\left(e^{j \omega}\right) \mathbf{g}\right|^{2}
$$

where

$$
\boldsymbol{\Phi}_{m, l}(z)=\phi\left(z W_{M}^{m} W_{D}^{l}\right) \phi^{T}\left(z W_{M}^{m}\right) .
$$

The output aliasing error is defined as

$$
\varepsilon_{D}=\frac{1}{2 \pi} \int_{-\pi}^{\pi} D\left(e^{j \omega}\right) d \omega=\mathbf{g}^{T} \mathbf{P g}
$$

where

$$
\mathbf{P}=\frac{1}{2 \pi D} \sum_{l=1}^{D-1} \sum_{m=0}^{M-1} \int_{-\pi}^{\pi} \boldsymbol{\Phi}_{m, l}^{H}\left(e^{j \omega}\right) \mathbf{h}^{*} \mathbf{h}^{T} \boldsymbol{\Phi}_{m, l}\left(e^{j \omega}\right) d \omega .
$$

The optimal synthesis prototype filter in terms of minimal system response error and minimal energy in the output aliasing terms is found by minimizing the total error function

$$
\begin{aligned}
\varepsilon_{t o t}(\mathbf{g}) & =\varepsilon_{T}(\mathbf{g})+\varepsilon_{D}(\mathbf{g}) \\
& =\mathbf{g}^{T}(\mathbf{E}+\mathbf{P}) \mathbf{g}-2 \mathbf{g}^{T} \mathbf{f}+1
\end{aligned}
$$

that is, through solving the linear equation system

$$
\mathbf{g}=\arg \min _{\mathbf{g}} \varepsilon_{t o t}(\mathbf{g})=(\mathbf{E}+\mathbf{P})^{-1} \mathbf{f}
$$

\section{EVALUATION}

We have designed two critically $(D=M)$ and two oversampled $\left(D=\frac{1}{2} M\right)$ decimated filter banks with 64 subbands and prototype analysis and synthesis filter lengths $L_{h}=L_{g}=128$. The decimation factor is set to $D=64$ or $D=32$ and the group delay is specified as $\tau_{d}=128$ or $\tau_{d}=64$, which gives four scenarios in total. The group delay of the prototype analysis filter is set to $\tau_{h}=\frac{1}{2} \tau_{d}$. Table 1 shows the filter bank performance measures after optimization, for the four scenarios. In this table, the System Phase Error is defined as

$$
\epsilon=\frac{1}{2 \pi} \int_{-\pi}^{\pi}\left|\angle T\left(e^{j \omega}\right)-\angle T\left(e^{j 0}\right)+\tau_{d} \omega\right| d \omega .
$$

We evaluate the performance of the filter banks in the case of a subband RLS beamformer with real data recorded in a hands-free car situation, $[7,8]$. In this situation we have a target signal, an interference signal, causing echo at the far end of the communication link, and background noise, see Fig. 2.

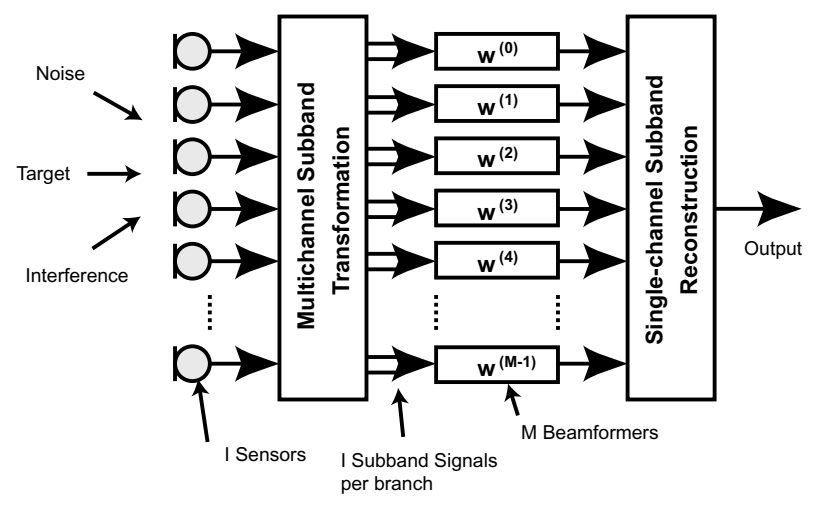

Figure 2: Subband FIR Beamforming Structure.

A linear array of six microphones is used and was mounted in a car on the visor at the passenger side. The distance between the speaker position and the microphone array is 350 $\mathrm{mm}$ and the position is perpendicular to the array axis at the center point. The spacing between adjacent elements in the array is $50 \mathrm{~mm}$. A known white noise sequence is emitted from a human shaped doll in order to determine the LS beamformer weights. The noisy background is recorded separately from the target and interference signals, in a car running at $110 \mathrm{~km} / \mathrm{h}$ on a normal asphalt road. Recordings 
of the background signal, the target signal and the interference signal serve as evaluation signals for the beamformer performance.

In order to measure the performance of the beamformer, we introduce the normalized distortion quantity

$$
\mathcal{D}=\frac{1}{2 \pi} \int_{-\pi}^{\pi}\left|C_{d} \hat{P}_{y_{S}}(\omega)-\hat{P}_{x_{S}}(\omega)\right| d \omega
$$

with

$$
C_{d}=\frac{\int_{-\pi}^{\pi} \hat{P}_{x_{S}}(\omega) d \omega}{\int_{-\pi}^{\pi} \hat{P}_{y_{S}}(\omega) d \omega}
$$

the normalized interference and noise suppression

$$
\mathcal{S}_{I}=\frac{\int_{-\pi}^{\pi} \hat{P}_{y_{I}}(\omega) d \omega}{C_{d} \int_{-\pi}^{\pi} \hat{P}_{x_{I}}(\omega) d \omega}, \quad \mathcal{S}_{N}=\frac{\int_{-\pi}^{\pi} \hat{P}_{y_{N}}(\omega) d \omega}{C_{d} \int_{-\pi}^{\pi} \hat{P}_{x_{N}}(\omega) d \omega} .
$$

Here, $\hat{P}_{x}$ is a PSD estimate of a single sensor observation and $\hat{P}_{y}$ is a PSD estimate of the beamformer output. The indices $S, I$ and $N$ denote the target speech component, the interference component and the noise component, respectively. The beamformer performance measures for the four scenarios are presented in Table 2. The results show that the beamformer performs better when oversampling is applied instead of critical sampling. They also show that the delays caused by the filter banks can be reduced at the expence of a minor deterioration of the beamformer performance. Fig. 3 shows short-time power estimates of the reference microphone signal and the beamformer output signal for the subband beamformer in scenario 3. We can observe that the background noise is suppressed by about $13 \mathrm{~dB}$ and that the interference signal (the male speaker) is suppressed by about $12 \mathrm{~dB}$.

\section{CONCLUSIONS}

We have proposed an efficient design method for a uniform DFT filter bank with the possibility of a pre-specified filter bank group delay. The method minimizes the inband and output aliasing error as well as the overall filter-bank transfer function's phase and amplitude deviation. The evaluation on a subband beamformer shows that the accuracy is dependent on both the group delay and the aliasing effects. Subband oversampling allows for a decrease in aliasing and amplitude errors, which in turn increases the performance significantly.

\section{REFERENCES}

[1] W. Kellermann, "Analysis and design of multirate systems for cancellation of acoustic echoes," in Proc. ICASSP'88, pp. 2570-2573.

[2] S. Haykin, "Adaptive Filter Theory," Prentice Hall Int. Inc., 1996, ISBN 0-13-397985-7.

[3] P. P. Vaidyanathan, "Orthonormal and biorthonormal filter banks as convolvers, and convolutional coding gain," IEEE Trans. on Signal Processing, vol. 41, no. 6, pp. 2110-2130, Jun. 1993.

[4] P. P. Vaidyanathan, "Multirate Systems and filter banks," Prentice Hall, 1993.

\begin{tabular}{r||c|c|c|c}
\hline \hline Case & $\varepsilon_{D_{0}}$ & $\varepsilon_{D}$ & $\varepsilon_{T}$ & $\epsilon$ \\
\hline \hline 1 & -51.3220 & -9.5093 & -6.6266 & 0.0393 \\
2 & -50.2648 & -8.9925 & -3.1576 & 0.0718 \\
\hline 3 & -71.8347 & -28.9326 & -23.8421 & 0.0022 \\
4 & -58.0498 & -23.3649 & -19.9155 & 0.0239 \\
\hline \hline & $\mathrm{dB}$ & $\mathrm{dB}$ & $\mathrm{dB}$ & $\mathrm{rad}$ \\
\hline \hline
\end{tabular}

Table 1: Filter bank performance for the four filter bank cases in the evaluation. The table shows the inband aliasing error $\varepsilon_{D_{0}}$, the output aliasing error $\varepsilon_{D}$, the system response error $\varepsilon_{T}$ and the system phase error $\epsilon$.

\begin{tabular}{r||l||r|r|r}
\hline \hline Case & $L=128, M=64$ & \multicolumn{1}{|c|}{$\mathcal{S}_{N}$} & \multicolumn{1}{|c|}{$\mathcal{S}_{I}$} & \multicolumn{1}{c}{$\mathcal{D}$} \\
\hline \hline 1 & $D=64, \tau_{d}=128$ & 9.4410 & 9.6890 & -22.9364 \\
2 & $D=64, \tau_{d}=64$ & 5.8847 & 8.5513 & -22.3555 \\
\hline 3 & $D=32, \tau_{d}=128$ & 13.0571 & 12.9027 & -28.3018 \\
4 & $D=32, \tau_{d}=64$ & 12.0605 & 12.6365 & -26.6816 \\
\hline \hline
\end{tabular}

Table 2: Performance measures for beamformer output in relation to the reference microphone input, when each signal component, speech, noise and echo components are active individually.

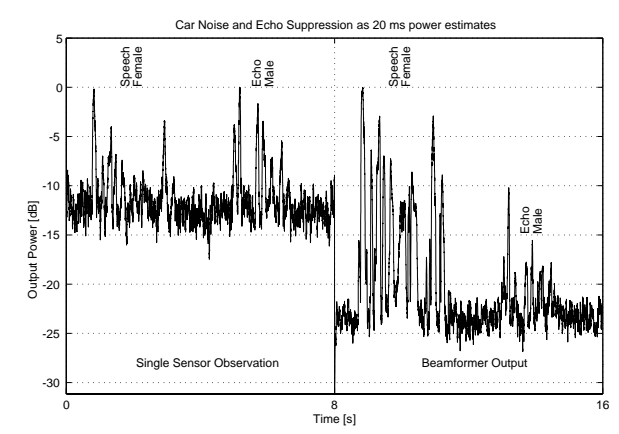

Figure 3: Short time (20 ms) power estimates of the reference microphone input signal (left) and the beamformer output signal (right). The figure corresponds to scenario 3 in the evaluation.

[5] A. Gilloire, M. Vetterli, "Adaptive filtering in subbands with critical sampling: analysis, experiments, and application to acoustic echo cancellation," IEEE Transactions on Signal Processing, pp. 1862-1875, vol. 40, issue 8, Aug. 1992.

[6] D. R Morgan, J. C. Thi, "A delayless subband adaptive filter architecture," IEEE Trans. Signal Processing, vol. 43, pp. 1819-1830, 1995.

[7] M. Dahl, I. Claesson "Acoustic Noise and Echo Cancelling with Microphone Array," IEEE Trans. on Vehicular Technology, vol. 48, No. 5, pp. 1518-1526, Sep. 1999.

[8] N. Grbić, "Speech Signal Extraction - A Multichannel Approach," University of Karlskrona/Ronneby, pp. 7275, Nov. 1999, ISBN 91-630-8841-X. 